\title{
Normalwerte von Proteinfraktionen im Lumballiquor und deren Quotienten nach Auftrennung in der Agar-Elektrophorese
}

\author{
Von H. P. RIEDer, P. R. Jung und V. Burrt \\ Aus dem Forscbungslaboratorium der Neurologischen Universitätsklinik Basel (Direktor: Prof. Dr. H. E. Kaeser)
}

(Eingegangen am 1. April/16. Juni 1972)

1. An einem Kollektiv von 53 retrospektiv ermittelten „Normalkontrollen“ wurden die Normbereiche der Proteinfraktionen nach elcktrophoretischer Auftrennung im Agargel überprüft. Die Bereiche werden verglichen mit den Werten anderer Autoren, welche nach dem gleichen methodischen Prinzip arbeiten, wie auch mit Proteinfraktionen von Normalen, die auf Celluloseacetatfolien getrennt wurden. Einige auffällige Unterschiede werden diskutiert.

2. Zusätzlich wurden die Quotienten Albumin/Globulin, Präalbumin/Albumin, $\alpha_{1}-/ \alpha_{2}$-Globuline, $\gamma$ - $/ \beta_{1}$-Globuline und $\gamma$-Globuline im Lumballiquor $/ \gamma$-Globuline im Serum berechnet und deren Normalbereiche bestimmt.

3. Es besteht keine gesicherte Geschlechtsabhängigkeit der Proteinfraktionen.

4. Mögliche Veränderungen von Fraktionen und Quotienten bei bestimmten Krankheitsprozessen und dic daraus resulticrende diagnostische Bedeutung von Elektrophoresedaten werden diskutiert.

\section{Determination of the normal concentrations and quotients for tilse protein fractions of lumbar cerebrospinal fuid by agar gel electrophoresis}

1. The proteins in the cerebrospinal fluid from 53 normal persons were separated by high voltage microelectrophoresis and their normal ranges were determined. The values are compared with those reported by other authors using similar methods, or electrophoresis on cellulose acetate. Marked differences between the results are discussed.

2. In addition, the normal ranges of the following quotients were computed: albumin/globulin, prealbumin/albumin, $\alpha_{1}-/ \alpha_{2}$-globulins, $\gamma$ - $/ \beta_{1}$-globulins, and $\gamma$-globulins in the lumbar cerebrospinal fluid $/ \gamma$-globulins in the serum.

3. No sex-linked variations in the distribution of the protein fractions could be found.

4. Possible changes in the concentration of fractions and in the quotients in neurological discases and their significance are discussed.

Jede Modifikation der Zonen-Elektrophorese, sei es hinsichtlich Trägermaterial (Papier, Agar, Celluloseacetatfolie etc.), sei es hinsichtlich Färbematerial (Amidoschwarz, Ponceaurot etc.) oder anderer Faktoren, führt $z u$ veränderten Relativprozent-Werten der aufgetrennten Proteinfraktionen. Normalbereiche können daher nicht einfach aus der Literatur übernommen werden; sie müssen für jede technische Variante neu erarbeitet werden.

Eine verbreitete Variante mit hoher Trennwirkung vor allem im $\gamma$-Globulinbereich ist die schon mehrfach beschriebene Mikroelektrophorese im Agargel (1-5), zu deren Gunsten in vielen Laboratorien die weniger aussagekräftige Papierelektrophorese verlassen worden ist. Daneben gewinnt heute trotz ihrer nicht ganz vergleichbaren Trennwirkung die einfacher $z u$ handhabende Elektrophorese auf Celluloseacetatfolien vermehrte Bedeutung.

$\mathrm{Da}$ das Erarbeiten von Normalbereichen der Proteinfraktionen im Liquor eine meistens nur retrospektiv zu verwirklichende und daher sehr zeitaufwendige Angelegenheit ist, möchten wir unsere über die letzten 5 Jahre zurückgreifenden Untersuchungen an „Nor-. malen" mit Hilfe der Agar-Elektrophorese (6) hier mitteilen. Es bietet sich damit zugleich die Gelegenheit an, die Ergebnisse mit kürzlich erschienenen Arbeiten von anderen Autoren $\mathrm{zu}$ vergleichen, welche über ent- sprechende Untersuchungen mittels Celluloseacetatfolien berichten $(7,8)$. Außerdem haben wir diesen Anlaß benützt, um auch die Normalbereiche bestimmter Fraktions-Relationen zu erfassen, die in den letzten Jahren - vor allem im Rahmen der Multiple SkleroseForschung - von einigen Autoren bei der Auswertung von Liquorbefunden diskutiert worden sind.

\section{Methodik}

Die Trennung erfolgte mittels der Hochspannungs-Mikroelektrophorese im Agargel (1) mit variablem Zusatz von Agarose (5-20\%) je nach Eigenschaft des verwendeten Agars (Difco, purified Agar). Laufbedingungen: Ionenstärke $\mu=0,06 ; \mathrm{F}=$ etwa $25 \mathrm{~V} / \mathrm{cm}$; Zcit: 26-27 min; Anfärbung mit Amidoschwarz $10 \mathrm{~B}$; Auswertung: bis 1969 mit dem Vitatron-Gerät nach dem unter (6) beschriebenen Prinzip, seither mit dem System Eppendorf-Densitometer mit $\mathbb{W}+\mathbb{W}$ Schreiber-Integrator, welches den großen Vorteil besitzt, die Albuminspitze ganz aufnehmen zu können, so daß eine rechnerische Ergänzung wegfällt. Die relative Farbstoffbindung der einzelnen Proteinfraktionen ist bekanntermaßen unterschiedlich. Die in rel-\% ausgedrückten Fraktionswerte müssen daher nicht notwendigerweise mit den absoluten Konzentrationsanteilen bestimmter Eiweiße übereinstimmen. Für die Beurteilung im klinischen Labor spiclt dies aber keine Rolle, sofern die zum Vergleich herangezogenen Normalbereiche unter den genau gleichen technischen Bedingungen ermittelt wurden. Die folgenden Mobilitätsbereiche wurden getrennt ausgewertet: Präalbumin, Albumin, die Globuline $\alpha_{1}, \alpha_{1}, \alpha_{2} \mathrm{M}, \alpha_{2} H, \beta_{1}, \beta_{2}, \tau$ sowie drei $\gamma$-Globulin-Subfraktionen: die relativ weitwandernden $\gamma_{0-2}\left(\gamma_{R}\right)$, die wenig bis nicht-wandernden $\gamma_{3-5}\left(\gamma_{\mathrm{B}}\right)$ und die kathodisch wandernden, also basischen Proteine $\gamma_{0-8}\left(\gamma_{0}\right)$. 
Tab. 1

Methodische Streuung: Agar-Elektrophorese

$\begin{array}{llllllll}\text { Alb } & \alpha_{1} & \alpha_{2} M & \alpha_{2} H & \beta_{1} & \gamma_{0-2} & \gamma_{3-8} & \gamma \text { tot }\end{array}$

$\begin{array}{lllllllll}\text { Mittelwert } * \overline{\mathrm{x}}[\text { rel-\%] } & \mathbf{7 3 , 3 4} & 4,55 & 3,25 & 4,15 & 5,30 & 3,28 & 4,33 & 7,61\end{array}$

Streuung s [rel- \%]

in der Serie:

von Tag zu Tag:

Variations-

koeffizient: [\%]

in der Serie : $\begin{array}{llllllll}1,27 & 0,27 & 0,47 & 0,52 & 0,44 & 0,36 & 0,29 & 0,45 \\ 1,52 & 0,60 & 0,49 & 0,88 & 0,57 & 0,38 & 0,56 & 0,62\end{array}$

von Tag zu Tag:

$\begin{array}{llllllll}1,8 & 6,0 & 14,4 & 12,5 & 8,3 & 11,0 & 6,7 & 5,9\end{array}$

$\begin{array}{llllllll}2,1 & 13,2 & 15,0 & 21,2 & 10,8 & 11,6 & 12,9 & 8,2\end{array}$

* die kleine Füllfraktion $\alpha_{i}$ und die beim Lagern nicht haltbare Fraktion $\beta_{2}$ bleiben unberücksichtigt; Präalbumin ist im Serum nur in Spuren vorhanden. Alle Auswertungen wurden von derselben Person ausgeführt

$\mathrm{Da}_{\mathrm{a}}$ im Einzelfall nur relativ geringe Liquormenger zur Verfügung stehen und die Liquorproteine bei längerem Aufbewahren unkontrollierbare Veränderungen aufweisen, ist uns die Abschätzung der rein metbodiscben Streunng, ,in der Serie“ und, "von Tag zu Tag“" nur aufgrund von analogen Reihenversuchen mit Kontrollseren möglich. Die Ergebnisse von 18 Doppelversuchen und 28 fortlaufenden Tagesversuchen mit demselben Serum (Roche) sind aus Tabelle 1 ersichtlich. Man erkennt, daß die große Albuminfraktion mit einem Variationskoeffizienten von rund $2 \%$ reproduzierbar ist, während die wichtigeren Globulinfraktionen VK zwischen $5-12 \%$ (Serie) aufweisen. Der Koeffizient von „Tag zu Tag“ ist im vorliegeriden Fall etwa anderthalb mal so groß, kann vereinzelt aber auch das Doppelte betragen. In Anbetracht der großen Unterteilung in viele, relativ kleine Fraktionen sind diese Werte befriedigend.

\section{Versuchspersonen}

Die Kriterien, die von uns zur Auswahl eines „Normalkollektivs“ gewählt wurden, sind bereits andernorts publiziert (9); der Kürze halber seien hier nur die wesentlichsten Erkrankungen erwähnt, welche neben den entzündlichen Prozessen des zentralen und peripheren Nervensystems ebenfalls ausgeschlossen wurden: Discopathien, Diabetes, Tumoren, Epilepsie nach kürzlichen Anfällen, Schizophrenie sowie. Patienten mit vorangegangener Luftencephalographie oder Myelographie etc.

In der Normalgruppe verbleiben somit vorwiegend Patienten mit Psychosen, funktionellen Störungen, Cephalaea, Migräne und andere Abklärungsfälle mit organisch negativem Befund und einige internmedizinische Patienten. Bei allen jedoch ist Voraussetzung, daß Zellzahl, PANDY-Test, Kolloidkurve, Totalprotein (bis $410 \mathrm{mg} / \mathrm{l}$ ) und eventuell andere klinisch-chemische Tests (Glucose, Chlorid) im Rahmen der Norm liegen.
Aus etwa 3000 Elektrophoresen von Patienten der Jahre 1966 bis 1971 waren 53 ,Normalkontrollen“ zu gewinnen:

Gruppe A: Patienten, die nach biologischen und klinischen Gesichtspunkten als ,normal" zu bezeichnen sind $(\mathrm{N}=27$ ).

Gruppe B: Patienten mit biologisch normalen Werten (Zellen, Mastix, Totalprotein $<450 \mathrm{mg} / \mathrm{l}$ ), deren klinische Befunde jedóch gegen eine sichere Einordnung in Gruppe $A$ sprechen $(N=26)$. Zur Bestimmung des Quotienten $\gamma$-Globuline im Liquor/ $\gamma$-Globuline im Serum (10) steher 28 Werte dex Gruppen A und B zur Verfügung.

Von jedem der beiden Kollektive werden Mittelwert und Streuung bestimmt; die statistische Verteilung der Einzelwerte ist normal mit Ausnahme der $\gamma_{\mathrm{c}}$-Globulin-Fraktion. Die Grenzwerte sind mit \pm 2 Standardabweichungen vom Mittelwert berechnet. Höchster und tiefster Normwert umschließen somit $95 \%$ der Fälle.

\section{Ergebnisse}

Wir haben die Gruppen der weitgehend „Normalen“" (A) und der fraglich „Normalen“" (B) graphisch nebeneinander aufgezeichnet. Es zeigt sich, daß die Mittelwerte beider Gruppen eng beisammen liegen und da $\beta$ auch deren Streüungen fast identisch sind.

Zur Berechnung von Normalbereichen der einzelnen Fraktionen habẹn wir die beiden Gruppen dementsprechend vereinigt und gelangen so, nach Eliminierung von drei inhomogenen Extremwerten, zur Gesamtgruppe von 53 Patienten. Die erhaltenen Normalwerte sind in Tabelle 2 angegeben.

Einige Autoren messen bei der Bewertung von Elektrophorese-Ergebnissen neben den Fraktionsanteilen auch verschiedenen Fraktionrelationen eine gewisse Bedeutung bei; so ist z. B. seit Jahtzehnten der Quotient Albumin/Globulin in Gebrauch. Neuerdings wird bei gewissen Erkrankungen auch Wert gelegt auf das Verhältnis Prä-Albumin/Albumin (3), $\alpha_{1}-/ \alpha_{2}-$ Globuline (2), $\gamma$ - $/ \beta$-Globuline (11) und $\gamma$-Globuline im Liquor $/ \gamma$-Globuline im Serum (10).

Wir haben die Quotienten bei jedem der obigen $\mathrm{Pa}$ tienten einzeln berechnet, daraufhin die Gruppen $A$ und $B$ miteinander verglichen. Es zeigt sich, daß die beiden Gruppen $A$ und $B$ sich wiederum gleich verhalten und zur Berechnung der Normalwerte zusammengefaßt werden dürfen (Abb. 1).

Tab. 2

Liquorelektrophoresen bei Normalkontrollen: Protein-Fraktionen in Relativprozenten

\begin{tabular}{|c|c|c|c|c|}
\hline \multirow[b]{2}{*}{ Fraktion } & \multirow[b]{2}{*}{$\begin{array}{c}\overline{\mathbf{x}} \\
{[\mathrm{rel}-\%]}\end{array}$} & \multirow[b]{2}{*}{$\begin{array}{c}\bar{x}+2 \text { s Bereich } \\
{[\text { rel- \%] }}\end{array}$} & \multicolumn{2}{|c|}{ Vergleich } \\
\hline & & & $\begin{array}{c}\text { Gruppe A } \\
\text { [rel-\%] }\end{array}$ & $\begin{array}{c}\text { Gruppe B } \\
\text { [rel-\%] }\end{array}$ \\
\hline Präalbumin & 6,0 & $3,6-8,4$ & $4,6-7,0$ & $3,4-8,8$ \\
\hline Albumin & 64,3 & $55,8-73.0$ & $56,3-73,0$ & $55,3-73,1$ \\
\hline$\alpha_{2}$-Globulin & 5,1 & $3,0-7,2$ & $3,3-7,3$ & $2,7-7,1$ \\
\hline$\alpha_{i}$-Globulin & 2,0 & $0,9-3,1$ & $0,9-3,3$ & $1,0-3,0$ \\
\hline$\alpha_{2} M-G l o b u l i n$ & 2,7 & $1,5-3,9$ & $1,6-4,0$ & $1,6-3,6$ \\
\hline$\alpha_{2} \mathrm{H}-\mathrm{Globulin}$ & 4,8 & $2,8-6,8$ & $2,5-7,3$ & $3,2-6,4$ \\
\hline$\beta_{1}$-Globulin & 5,9 & $4,0-7,8$ & $3,8-7,6$ & $4,3-7,9$ \\
\hline$\beta_{2}$-Globulin & 1,8 & $0,8-2,8$ & $1,6-2,8$ & $1,0-2,6$ \\
\hline$\tau$-Globulin & 1,7 & $0,9-2,5$ & $0,9-2,6$ & $1,2-2,4$ \\
\hline$\gamma_{0-2}$-Globulin & 3,1 & $1,5-4,6$ & $1,2=4,3$ & $1,8-4,7$ \\
\hline$\gamma_{3-6}$-Globulin & 1,7 & $0,6-2,6$ & $0,5-2,5$ & $0,7-2,7$ \\
\hline$y_{0--G l o b u l i n}$ & 0,3 & $0,0-0,9 *$ & $0,0-0,9 *$ & $0,0-0,9 *$ \\
\hline
\end{tabular}

* Wegen der asymmetrischen Verteilung von $\gamma_{\text {.- }}$ gelten Werte bis zu $0,9 \%$ als "Normal" 


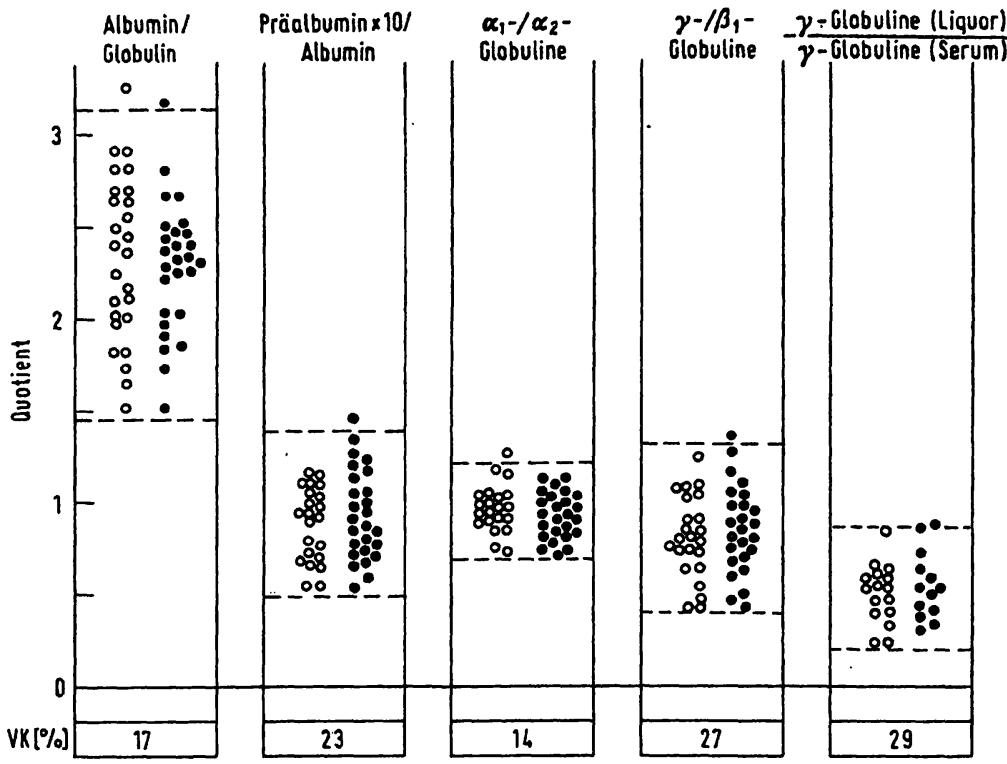

Abb. 1

Liquor-Protein-Elektrophorese Quotienten einiger Liquorfraktionen (rel-\%/rel- \% = Gruppe A: weitgehend Normale"

- = Gruppe B: fraglich Normale"

VK $=$ Biologischer Variationskoeffizient der verschiedenen Quotienten in Prozent

Abb. 2

Agargel-Elektrophorese (nach WIEME) Von Liquores weitGehend "normaler Patienten" tive" (rel-or "NormalkollekLÖWENTHAL (2), 1 ( $2 \mathrm{~s}$ ) von: SOWENTHAL SCHMI, (5) mit nissen dieser Arbeit (Gruppe $A$ und $B$ )

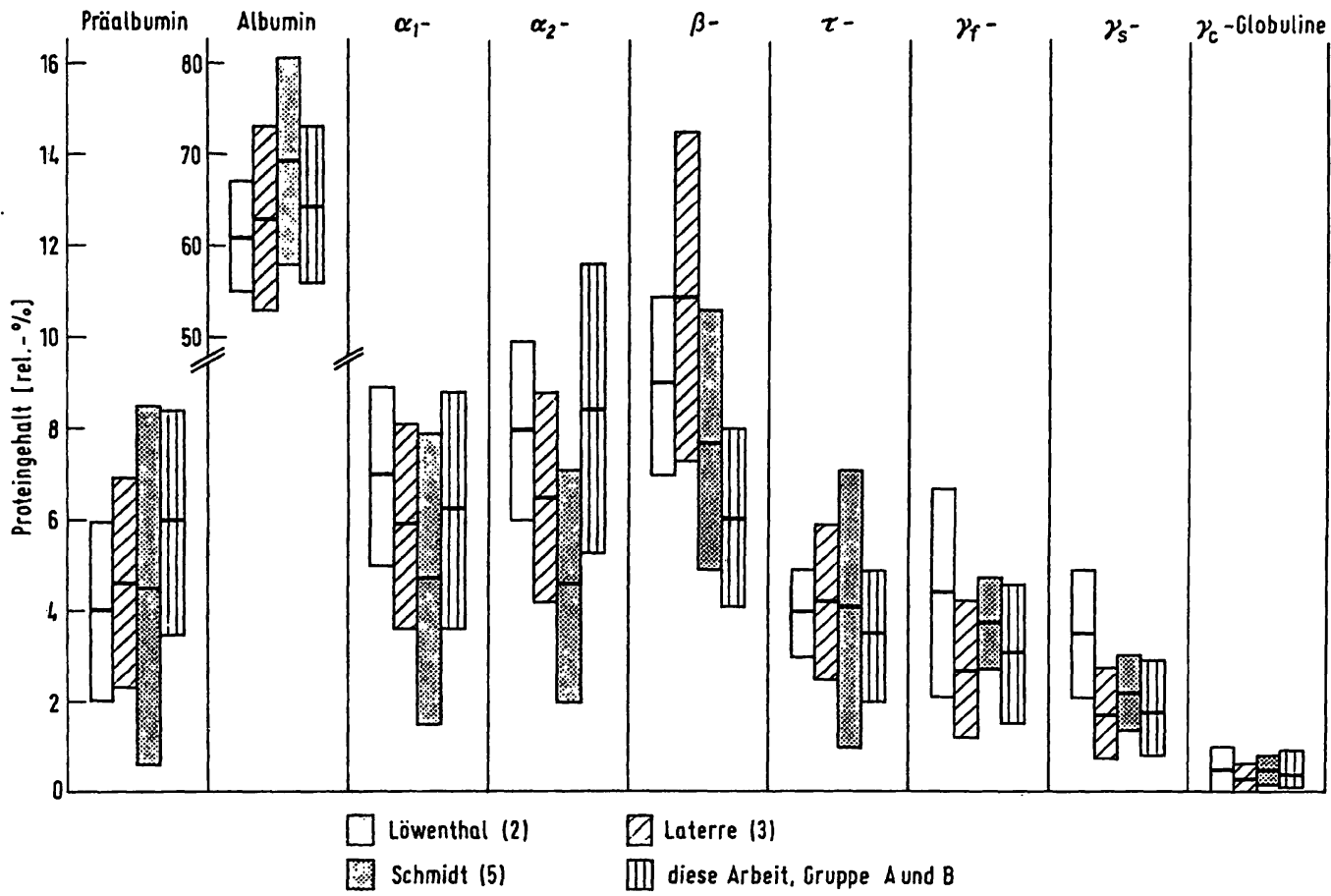

Tab. 3

Normalbereiche der Quotienten bestimmter LiquorproteinFraktionen

\begin{tabular}{|c|c|c|c|}
\hline Quotient & $\begin{array}{c}\overline{\mathbf{x}} \\
{[\text { rel-\%] }} \\
\text { rel-\%] }\end{array}$ & $\begin{array}{c}\overline{\mathbf{x}}+\text { 2s Bereich } \\
{[\text { rel-\%/rel-\%] }}\end{array}$ & VK in $\%$ \\
\hline Albumin/Globulin & 2,39 & $1,45-3,13$ & 17 \\
\hline Präalbumin/Albumin & 0,93 & $0,50-1,37$ & 24 \\
\hline$\alpha_{1}-/ \alpha_{2}-$ Globulin & 0,95 & $0,69-1,25$ & 14 \\
\hline$\gamma-/ \beta_{1}$-Globulin & 0,85 & $0,39-1,31$ & 27 \\
\hline $\begin{array}{c}\gamma \text {-Globulin } \\
\text { Liquor/Serum }\end{array}$ & 0,53 & $0,21-0,85$ & 29 \\
\hline
\end{tabular}

Die erhaltenen Normalbereiche der Quotienten sind in Tabelle 3 angegeben. In Abbildung 2 werden die auf weitgehend ähnliche Art gewonnenen Normalbereiche von Lö WENTHAL (2), LatrRre (3), Schmidt (5) und von uns miteinander verglichen. Ferner gibt Tabelle 4 Auskunft über die Unterschiede zwischen der Celluloseacetat- und der Agargel-Technik in Form einer Gegenüberstellung unserer Werte mit denjenigen von MERTIN et al. (7) und Castaigne et al. (8).

\section{Diskussion}

Beim Vergleich unserer Normalberciche der LiquorFraktionen mit denen der Serumprotein-Fraktionen (6) fallen erwartungsgemäß die hohen Präalbuminwerte, die niedrigen $\gamma$-Globulin-Werte, die zusätzliche $\tau$-Globulin-Fraktion und die flache Verteilung der $\alpha_{2}$-Globuline im Liquor auf, welche ja zum Ausdruck bringen, daß Liquor nicht nur ein einfaches Serumfiltrat darstellt. Die von Schwartz et al. (12) früher in diesem Labor verwendeten Normalgrenzen der $\gamma$-Globuline stimmen 
Tab. 4

Vergleich von "Normalwerten" des Liquorproteins nach elektrophoretischer Fraktionierung auf Celluloseacetatfolien und im Agargel bei übereinstimmender Anfärbung mit Amidoschwarz

\begin{tabular}{|c|c|c|c|c|c|c|c|}
\hline $\begin{array}{l}\text { Probandenkollektiv } \\
\text { und Trägermedium }\end{array}$ & Präalbumin & Albumin & $\begin{array}{c}\text { Elektrophoretische } \\
\alpha_{1} \text {-Globulin }\end{array}$ & $\begin{array}{l}\text { Fraktionen: } \bar{x} \\
\alpha_{2}-\text { Globulin }\end{array}$ & $\begin{array}{l}\text { nd } \bar{x} \pm 2 \text { s [re } \\
\beta_{1} \text {-Globulin }\end{array}$ & $\tau$-Globulin & $\gamma$-Globulin \\
\hline $\begin{array}{c}\text { Agar: } \\
\text { diese Arbeit: } \\
(\mathrm{N}=53)\end{array}$ & $\begin{array}{c}6,0 \\
3,5-8,4\end{array}$ & $\begin{array}{c}64,3 \\
55,8-73,0\end{array}$ & $\begin{array}{c}6,2 \\
3,6-8,8\end{array}$ & $\begin{array}{c}8,4 \\
5,3-11,6\end{array}$ & $4,0-7,8$ & $2,0-^{3,5} 4,9$ & $\stackrel{5,1}{2,3-8,0}$ \\
\hline $\begin{array}{l}\text { Cellulose-Acetat-Folie: } \\
\text { MERTIN et al. ( } 7) \\
(N=104) \\
\text { CASTAIGNE et al. (8) } \\
((N=28) \\
\gamma \text {-Globuline, chemisch: }\end{array}$ & $\begin{array}{l}2,5-7,9 \\
6,1 \\
1,7-10,5\end{array}$ & $\begin{array}{c}62,7 \\
53,0-72,4 \\
58,5 \\
45,0-72,0\end{array}$ & $\begin{array}{l}3,6 \\
1,1-6,1 \\
4,5 \\
1,6-7,4\end{array}$ & $\begin{array}{l}5,0 \\
2,7-7,3 \\
4,6 \\
1,8-7,4\end{array}$ & $\begin{array}{c}8,8 \\
6,2-11,5 \\
10,3 \\
3,9-16,7\end{array}$ & $\begin{array}{c}6,1 \\
2,8-9,3 \\
\begin{array}{c}6,2 \\
1,3-11,1\end{array}\end{array}$ & $\begin{array}{c}8,6 \\
4,0-13,2 \\
9,5 \\
4,7-14,3 \\
5,1 \\
1,9-8,3\end{array}$ \\
\hline
\end{tabular}

* Position des Auftrags

+ Die Fraktion $\alpha_{1}$ der Agar-Elektrophorese wurde zu Vergleichszwecken je hälftig auf die Fraktionen $\alpha_{1}$ und $\alpha_{2}$ verteilt

weitgehend mit den vorliegenden Ergebnissen überein.

$\mathrm{Zu}$ Vergleichszwecken bieten sich die Untersuchungen von Löwenthal (2), von LATERre (3) sowie von Schimdi (5) an, die mit dem gleichen Agar-Elektrophoresesystem arbeiten (1). Als erstes muß hier allerdings festgehalten werden, daß die von LöwENTHAL beschriebene ' $\tau$-Globulin-Fraktion mit verminderter Mobilität (gegenüber der entsprechenden $\beta_{2}$-GlobulinFraktion des Serums) unter unseren sehr ähnlichen Arbeitsbedingungen nicht mit der exwarteten Regelmäßigkeit und Deutlichkeit von $\operatorname{der} \beta_{2}$-Position unterschieden werden kann. In seltenen Fällen ist eine Doppellinie zu erkennen, welche etwa den Positionen $\operatorname{der} \beta_{2^{-}}$ und $\tau$-Globuline entsprechen könnte; meistens jedoch ist nur ein einzelner, aus beiden Fraktionen zusammengesetzter Peak mit mittlerer Wanderungscharakteristik zu erkennen. Ob dies am Agarosezusatz zum Trägermedium oder an der Liquorkonzentrierung in der Kälte liegt, vermögen wir nicht mit Sicherheit zu entscheiden.

Abbildung 2 vermittelt einen Überblick über die mit der Agarelektrophorese gefundenen Verhältnisse bei „Normalen". Obwohl im großen und ganzen eine vernünftige Übereinstimmung des Trennbildes besteht, zeigt die Abbildung doch auch das mögliche Ausma $B$ von $A b-$ weichungen, die wahrscheinlich auf geringfügigen Unterschieden in der Auswertetechnik und offenbar nicht ganz gleichwertigen "Normalkollektiven" beruhen. So fällt das Kollektiv von ScHmid nicht nur durch einige stark abweichende Mittelwerte einzelner Fraktionen (hohe Albumin- und sehr niedrige $\alpha_{1}$ - und $\alpha_{2}-$ Globuline-Werte) auf, sondern auch durch vereinzelt übermäßig große Streubereiche (Präalbumin, $\alpha_{1}$ - und $\tau$-Globuline). Der Schluß liegt nahe, daß es sich hier um ein allzu inhomogenes Vergleichsgut handelt, in welchem vermutlich Fälle mit leichten Permeabilitätsstörungen mit ihrer Präalbumin/Albumin-Dissoziation, Verdünnung der $\tau$-Globuline und eventueller Beeinträchtigung der $\alpha_{1}$-Globuline nicht selten sind. Auch beim Kollektiv von LöWENTHAL scheint der sehr̉ niedrige Mittelwert für Präalbumin und der auf der anderen
Seite relativ hohe $\boldsymbol{\gamma}$-Globulingehalt auf Permeabilitätsstörungen vom Plasmatyp hinzuweisen, die nach unseren Erfahrungen z. B. auch bei peripher-nervösen Erkrankungen oder mechanischen Schäden wie Diskushernien nicht selten anzutreffen sind. Außerdem geht aus den Angaben von LöweNTHAL nicht ganz eindeutig hervor, $\mathrm{ob}$ in seinen Tabellen die gesamte Albuminftaktion gezählt, oder ob - aus apparativen Gründen - die oberste Spitze nicht miterfaßt ist, so daß die Globulinwerte an sich schon höher und damit nicht streng vergleichbar herauskämen. Am besten stimmen unsere eigenen Werte mit denjenigen von LATERRE überein; einzig im $\alpha_{2}-\beta$-Globulin-Bereich scheint LATERRE eine andere Unterteilung anzuwenden als wir. Aufgrund der Wert-Verhältnisse ist zu vermuten, $\mathrm{da} \beta$ er die zwischen $\operatorname{den} \alpha_{2}$ - und $\beta$-Globulin-Zonen liegenden Proteine zu der $\beta_{1}$-Globulin-Fraktion rechnet und damit zu einer auffällig hohen und breit streuenden $\beta$-Globulin-Fraktion gelangt. Jedenfalls kann dieser Vergleich als weiteres Beispiel dafür dienen, wie wichtig es selbst bei im Prinzip gleichartiger Technik ist, daß jedes Laboratorium die Normalbereiche anhand eigener Kontrollen überprüft. Beim Vergleich unserer Normalwerte der Agar-Elektrophorese mit denjenigen von Mertrn (7) oder CASTAAIGNE (8) auf Celluloseacetat springen einige nennenswerte Unterschiede ins Auge (vgl. Tab. 4). Obwohl natürlich eine strenge Vergleichbarkeit der "Normalkollektive“ nicht vorausgesetzt werden darf, scheint sich doch die schon mehrfach beobachtete Tatsache zu bestätigen, $\mathrm{da} B$ die Agar-Elektrophorese etwas höhere Albuminwerte liefert. Viel auffallender sind aber die Unterschiede im $\alpha_{1}-\alpha_{2}-$ Globulin-Bereich einerseits und dem $\beta-\gamma$-Globulin-Bereich anderseits, wo eine vollkommene Inversion der Werthöhen zu sehen ist.

Die ganz verschiedenen Eigenschaften des Celluloseacetat- und des Agargels bedingen unterschiedliche Auftragsorte der Probe auf der Trennbahn. Während bei der üblichen Routinetrennung im alkalischen Puffer bei der Celluloseacetatfolie alle Eiweiße in Richtung Anode vom Auftragsort wegwandern, bewirkt der saure Charakter des Agargels eine starke Elektroendosmose in Richtung Kathode, von welcher primär 

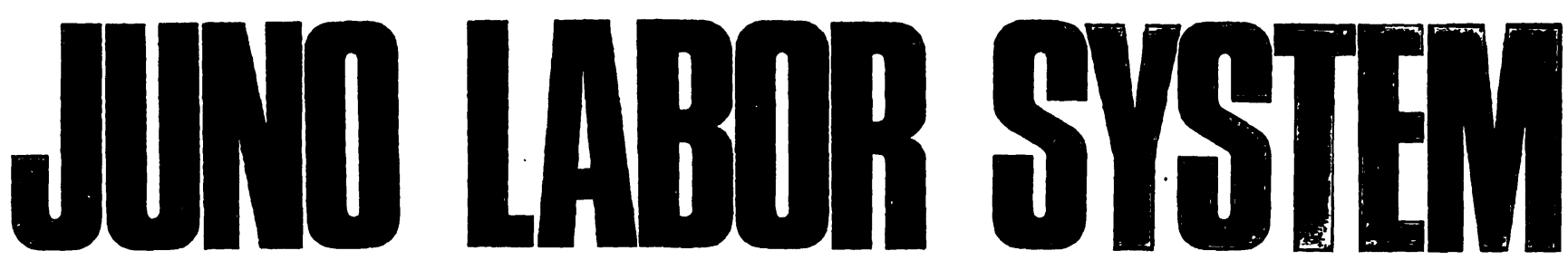

PROGRAMME $2001+2012$

Volle Versorgung mit allen gewünschten Medien ob Wandtisch oder Doppeltisch in Stehhöhe, Sitzhöhe, als Tieftisch verkleidete Energiezelle mit 1,5 mm starken Stahlblechen säurebeständige ACRYLHARZLackierung in RAL 7021 falls notwendig in PE-Beschichtung besser geht es nicht. -

Trennwände nur im Programm 2001 Füllungen ab $1.310 \mathrm{~mm}$ über OKF Die Füllungen in MELAMINHARZPlatten, Glas, Gips, Eternit wie Sie es brauchen die Raumhöhe ist uns egal die Deckenschiene schluckt die baulichen Toleranzen Vollinstallierte Trennwände sind eine JUNO-Leistung sie können sich sehen lassen nachträglich aufbaubar und zu versetzen immer aber frühzeitig zu planen Sie sollten sich entscheiden zwischen der gemauerten Wand und der vollinstallierten JUNO-Trennwand -

\section{Normgruppe Trennwände}

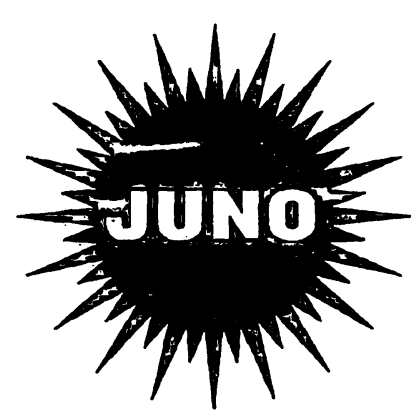

- - JUNO-REFERENZEN-INSTITUTSBAU - - STHB-ELLWANGEN-ING. SCHULE AALEN - - UNIVERSITÄT SAARBRÖCKEN - ORGANISCHE CHEMIE - - UNIVERSITÄT GIESSEN - PHYSIOLOGISCHE CHEMIE - - RUHRUNIVERSITÄT BOCHUM (ARGE) ALLE INSTITUTE DER NATURWISSENSCHAFTEN - - MEDIZINISCHE HOCHSCHULE - HANNOVER - FORSCHUNG - - KFA - JÜLICH - PHYSIKALISCHE CHEMIE - - TECHNISCHE HOCHSCHULE ZÜRICH - 


\section{BIOCHIMIE}

\section{Edité par la Société de Chimie Biologique}

tel est le titre

sous lequel paraîtra à partir de 1971

le „BULLETIN DE LA SOCIÉTÉ DE CHIMIE BIOLOGIQUE“ .,

\section{SECRÉTARIAT}

de la Société de Chimie Biologique

J. P. EBEL, Secrétaire Général (Relations Extérieures)

R. PERLES, Secrétaire Général

\section{REDACTION}

F. GROS, Secrétaire scientifique

F. PERCHERON, Secrétaire à la Publication $J$. NU'NEZ, Secrétaire à l'Information

Y. RAOUL, Secrétaire à l'Edition

SECRETARIAT et REDACTION: 4 Avenue de l'Observatoire, PARIS $6^{\circ}$

12 FASCICULES

ABONNEMENTS: FRANCE et ZONE FRANC: 150 ffres - BELGIQUE: 1.687, - frcs - AUTRES PAYS: $186,-$ ffrcs

MASSON et Cie, Editeurs - 120 Boulevard St Germain - PARIS 6ème

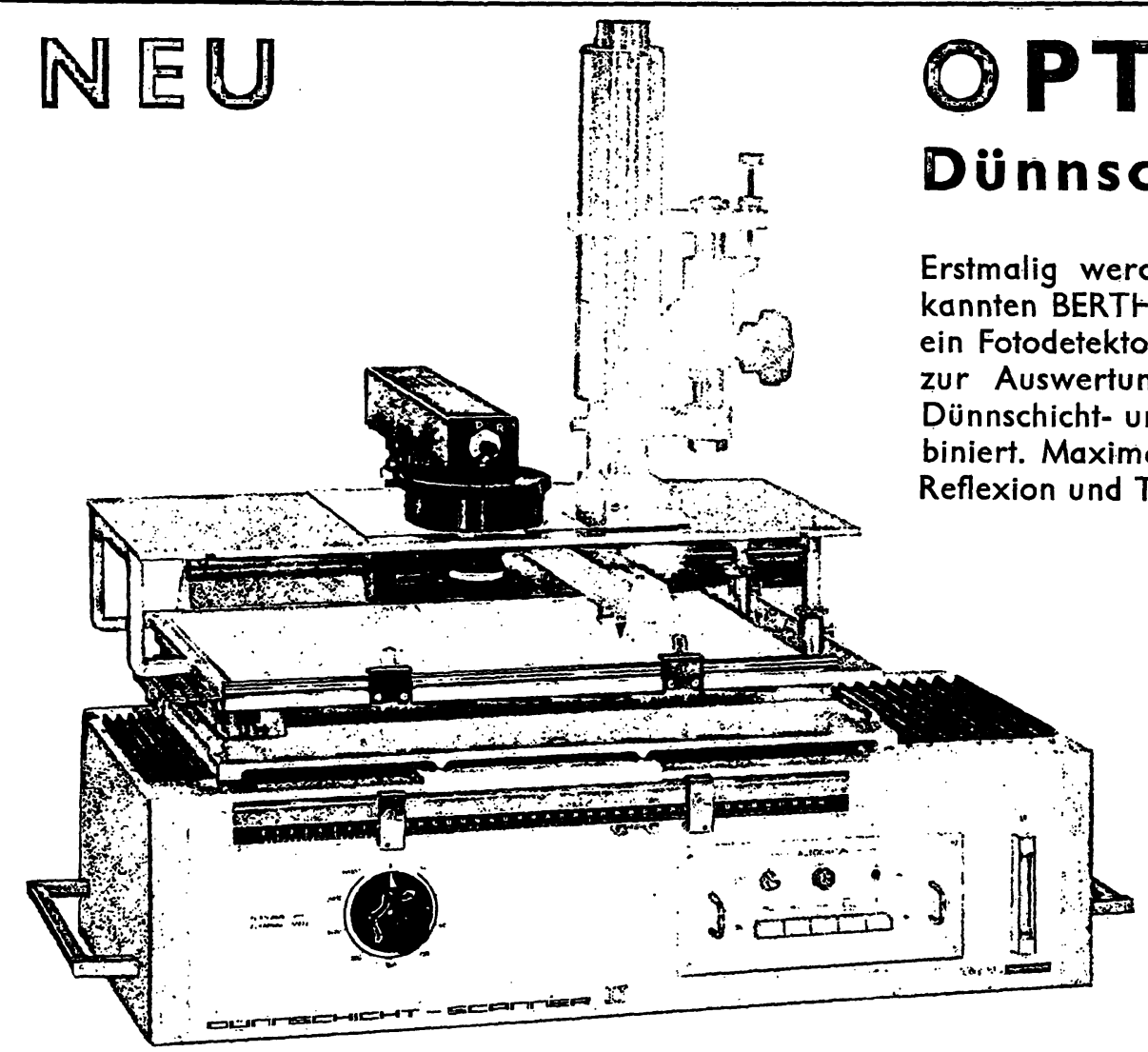

SCHER icht - Scanner

Erstmalig werden - aufbauend auf dem bekannten BERTHOLD-,,Dünnschichtscanner"II Fodetektor und ein Radioaktivitätsdetektor Dunnschicht- und Papierchromatogramme kombiniert. Maximale Abtastfläche $160 \times 400 \mathrm{~mm}$ in Reflexion und Transmission.

Hier nur einige Beispiele für die völlig neven Anwendungṣmöglichkeiten:

Automatische MultibandElektrophoresen von Serumproteinen auf CAF

Zerstörungsfreie Bestimmung der spezifischen Radioaktivität entlang der Chromatogrammstrecke Prüfung auf Farbstoffstabilität bzw. Ausbleichung.

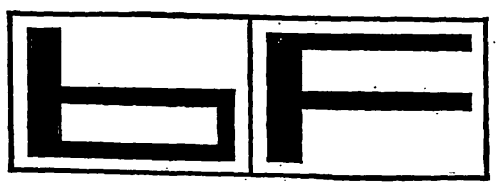

VERTRIEBS-GMBH FÜR MESSTECHNIK der Firmen Frieseke \& Hoepfner $\mathrm{GmbH}$. Erlangen-Bruck und-Laboratorium Prof. Dr. Rudolf Berthold - Wildbad

75 Karlsruhe 41 - Berwaldstraße 30 . Postfach 410220 - Telefon (07 21) $401011-14$ - Telex 7825928 . Telegramm Raylec 
alle Proteine um einen bestimmten Betrag kathodenwärts transportiert werden. Dieser endosmotischen Dislokation entgegengesetzt verläuft die eigentliche Wanderung im elektrischen Feld, so daß die tatsächliche Verschiebung eines bestimmten Eiweißes die Summe beider Wirkungsrichtungen repräsentiert. Um die Trennstrecke auf dem agarbeschichteten Objektträger optimal auszunützen, ist die Probe erfahrungsgemäß so zu applizieren, da $\beta$ der Auftragsschlitz $z$ wischen die Fraktionen der $\alpha_{2}-$ und $\beta_{1}$-Globuline zu liegen kommt.

Unter diesem Aspekt lassen sich die zwischen der Celluloseacetat- und der Agarelektrophorese gefundenen Unterschiede auch in dem Sinne beschreiben, daß offenbar die Zonen unmittelbar anodenwärts vom Auftragspunkt (Doppelstrich in Tab. 4) durch erhöhten Eiweißreichtum auffallen. Könnte es sich dabei eventuell um relativ große Proteine (nicht unbedingt Immunglobuline) handeln, die zwar eine starke elektrische Ladung aufweisen, deren tatsächliche Wanderung aber aus räumlichen Gründen nur gering ist? Die von CASTAIGNE et al. gleichzeitig ausgeführte chemische Bestimmung der $\gamma$-Globuline mit dem Ergebnis eines nur etwa halb so großen Wertes wie die Cellulose-Acetat-Folien-Elektrophorese vorgibt, könnte eine solche Vermutung stützen (vgl. Tab. 4). Oder haben wir in der unterschiedlichen optischen Dichte der genannten Zonen eine spezifische Einwirkung des Trägermediums auf die Anfärbbarkeit der $\alpha$-resp. $\beta$ - $\gamma$-Globuline zu sehen? Aufgrund unserer eigenen Erfahrungen ist diese Frage vorderhand nicht zu entscheiden.

Bei der Beurteilung von Elektrophoresen stellt sich nun die Frage, wieweit relative Verscbiebungen bestimmter Fraktionen und deren gesonderte Auswertung das übliche Elektrophoresebild zu ergänzen und die Aussage zu bereichern vermögen. Derartige Fraktionsveränderungen sind ja bei einer Vielzahl von Erkrankungen bekannt; ein kurzer Ubberblick soll die verschie denen Möglichkeiten nochmals vor Augen führen (4):

Bei Permeabilitätsstörungen nehmen die Präalbuminwerte des Liquors infolge Verdünnung durch Plasmaproteine $a b$, während die $\gamma$-Globuline meist zunehmen; das Bild gleicht sich demjenigen des Serums an (13). Beim Guillain-Barré-Syndrom, bei Tumoren, Meningitiden, cerebrovaskulären Prozessen u. a. ist diese Reaktionsform die übliche. Degenerative Prozesse andererseits lassen häufig das Präalbumin ansteigen.

Die Albrimin-Werte verhalten sich uneinheitlich. Bei den obgenannten Permeabilitätsstörungen können sie Relativprozent-Werte wie im Serum annehmen, bei geringer, erst die kleinen Proteine betreffender Störung aber auch darüberhinaus ansteigen; bei Degenerationserscheinungen und immunologischen Prozessen werden sie entsprechend kleiner oder bleiben unverändert.

$\alpha$-Globuline scheinen sich einerseits mit dem Grad von Entzündungen, anderseits auch bei metabolischen (Lipidosen, Arteriosklerose) und neoplastischen Prozessen in ihrer relativen Werthöhe zu verschieben.

Bei Degenerationen des Zentralnervensystems ist häufig ein Anstieg der $\tau$-Globuline zu beobachten, während die Gesamt- $\gamma$-Globulin-Werte eher niedriger werden. Diese letzteren nehmen, abgesehen von den genannten Permeabilitätsstörungen, bei akuten und chronischen Entzündungen zu.

Wir unterscheiden ein diffuses Ansteigen der Relativprozent-Werte von $\gamma$-Globulinen bei GurrLAIN-BARré-Syndrom oder Meningitis, eventuell auch bei Tumoren usw., und ein fraktioniertes, auch "diskontinuierlich“ oder „oligoclonal" genanntes Ansteigen einzelner $\boldsymbol{\gamma}$-Globulin-Zonen, wic etwa bci Multipler Sklerose, LeukoEnzephalitis und Neurosyphilis. Der Bereich der $\gamma$-Globuline im Liquor kann dann eine bei Normalen nie zu schende Aufspaltung in meist mehrere Paraprotcinbanden $(12,14,15,16)$ zeigen.

Aus allen diesen Beobachtungen ist $z u \cdot$ schließen, daß nicht nur die Werthöhe von Einzelfraktionen, sondern auch ihre Relationen klinisch wichtige Informationen erwarten lassen. Als Grundlage für Untersuchungen in dieser Richtung ist es vorerst nötig, die Streubereiche einiger ausgewählter Relationen bei Normalkontrollen zu kennen (vgl. Tab. 3).

Solche Relationen lassen sich gut anhand von Quotienten erfassen: so deutet ein veränderter Albumin/GlobulinQuotient auf einen Anstieg der Globuline und/oder Präalbumine hin, eventuell aber auch auf einen Albuminverlust z. B. bei Nierenschaden.

Niedere Präalbumin/Albumin-Quotienten findet man ganz allgemein bei Permeabilitätsstörungen (13), gelegentlich auch bei degenerativen Prozessen: HeredoDegenerationen, Cerebral-Atrophie.

Bei vielen neoplastischen Prozessen erhöht sich das Verhältnis $\alpha_{1}-/ \alpha_{2}$-Globuline (normalerweise sind die Werte der $\alpha_{2}$-Globuline ähnlich denen der $\alpha_{1}$-Globuline) $(3,15)$.

In ihren Untersuchungen an Multiple Sklerose-Patienten messen BERGMANN et al. (11) dem Verhältnis $\gamma / \beta$-Globuline einige Bedeutung zu. Sie geben als Norm 0,5 an; Werte über 0,75 sind nach ihnen als pathologisch $z u$ werten. Schwartz et al. legen ihre Normbereiche sehr ähnlich auf 0,3-0,8 fest (12). Bei 60-75\% der MultipleSklerose-Patienten finden sie einen Anstieg des $\gamma$ - $/ \beta$ Globulin-Quotienten. Bei degenerativen Prozessen ist er erniedrigt.

In der vorliegenden Studie haben wir die $\gamma$-GlobulinWerte mit den $\beta_{1}$-Globulin-Werten in Beziehung gesetzt, da sich in der Agargel-Elektrophorese die $\beta_{2}$ - und/oder $\tau$-Globulin-Fraktionen deutlich von $\operatorname{der} \beta_{1}$-GlobulinFraktion abtrennen lassen und die $\beta_{1}$-Globulin-Werte die größte Konstanz aufweisen (2). Dementsprechend erhalten wir höhere Normalbereiche: $\gamma-/ \beta_{1}$-Globuline $=0,4-1,3$.

Bei entzündlichen Erkrankungen des Zentralnervensystems, wie im besonderen bei der Multiplen Sklerose, dürfte auch der $\gamma$-Globulin-Quotient (Liquor/Serum) von Schinko und Tschabitscher zusätzliche Aussagen ermöglichen. Sie beobachten bei der Multiplen Sklerose einen Anstieg der Werte im ersten Krankheitsjahr $(10,11)$. Dabei sprechen Werte über 0,8 bei entsprechender Symptomatik für eine Multiple Sklerose (10). Unsere eigenen Normalwerte umfassen den Bereich von 0,21-0,85; die obere Grenze liegt demnach etwas höher als bei den vorhin genannten Autoren. (Dieses Kriterium ist insofern von beschränkterem Wert, als es von allen von uns untersuchten Quotienten den größten Variationskoeffizienten aufweist (VK = 30\%).)

Eine Steigerung der Aussagekapazität von Elektrophorese-Ergebnissen läßt sich nur durch Techniken mit wesentlich vermehrtem Arbeitsaufwand erreichen, der 
sich vorläufig einer routinemäßigen Anwendung im klinischen Laboratorium verbietet $(14,17)$. Nach wie vor sind deshalb die Ergebnisse der einfachen Agargel- oder Cellulose-Acetat-Folien-Elektrophorese unersetzlich bei der Diagnose der Leuko-Enzephalitis, Trypanosomiasis, der Neuro-Lues und vor allem der Multiplen Sklerose, wie auch bei der Abklärung manch anderer Fragestellungen (Tumoren, degenerative Prozesse, etc.). Infolge der relativen Häufigkeit dieser Krankheiten bleibt sie ein wichtiges Hilfsmittel der Diagnostik.

\section{Literatur}

1. Wieme, R. J. (1965), Agargel electrophoresis. Elsevier Publishing Company, Amsterdam. - 2. Löwentrial, A. (1964), Agargel electrophoresis in neurology. Elsevier Publishing Com= pany, Amsterdam. - 3. Laterre, E. CH. (1965), Les protéines du liquide céphalo-rachidien à l'état normal et pathologique. Edition Arscia S. A., Bruxelles. - 4. Schultze, E. H., Heremans, J. F. (1966), Molecular Biology of Human Proteins. Vol. I, pp. 182 and 222. Elsevier Publishing Company, Amsterdam, New York, London. - 5. Schmidt, R. M. (1968), Der Liquor cerebrospinalis. Verlag Volk und Gesundheit, Berlin. - 6. RIEDER, H. P., Nusselt, L. (1969), Clin. Chim. Acta 24, 159-170. - 7. Mertin, E., Wisser, H. \& Doerr, P. (1971), diese Z. 9, 337-340. - 8. Castaigne, P., L'Hermitte, F., Schuller, E., Rouques, C. \& LoRIDAN, M. (1971), Rev. Neurol. 124, 97-105. - 9. Jung, P. R.,
RIEDER, H. P. \& JeLtsCh, C. (1972), Klin. Wochenschr. im Druck. 10. Panagiotopoulos, J., Pernhaupt, G. \& Tschabitscher, $H$. (1964), Wien. Z. Nervenheilk. 18, Suppl. 1, 195-207. - 11. Bergmann, L., Gilland, O., Otyanders, S. \& Svennerholm, L. (1964), Acta Neurol. Scand. 40, Suppl. 10, 33-48. - 12. SchwarTz, S., RIEDER, H. P. \& Wüthrich, R. (1970), Europ. Neurol. 4, 267-282. - 13. RIEDER, H. P., KAESER, H. E. \& NuSSELT, L. (1972), Schweiz.Med. Wochenschr. 102, 766-772. - 14. MAURIN, P., Henry, P. \& Neuzin, E. (1970), Bordeaux médical 2, 299-307. 15. Rice, E. W. \& Loftrs, J. W. (1962), Clin. Chem. 8, 56-65. 16. BergmanN, L. (1965), Acta Neurol. Scand. 41, Suppl. 13, 559-561. - 17. Greenhouse, A. H. \& Speck, L. B. (1964), Amer. J. Med. Sci. 248, 333-340.
Dr. H. P. Rieder

Neurologische Universitätsklinik CH-4051 Basel 